\section{BRAZIULIAN JOURNAL \\ OF MIEDICAL AND BIOLOGICAL RESEARCH}

www.bjournal.com.br
ISSN 0100-879X

Volume 45 (6) 473-564 June 2012

BIOMEDICAL SCIENCES

AND

CLINICAL INVESTIGATION

Braz J Med Biol Res, May 2012, Volume 45(6) 546-556

doi: $10.1590 / \mathrm{S} 0100-879 X 2012007500056$

Analysis of acylcarnitine profiles in umbilical cord blood and during the early neonatal period by electrospray ionization tandem mass spectrometry

E. Vieira Neto, A.A. Fonseca, R.F. Almeida, M.P. Figueiredo, M.A.S. Porto and M.G. Ribeiro

The Brazilian Journal of Medical and Biological Research is partially financed by

\section{욛NPq}

da Ciência e Tecnologia

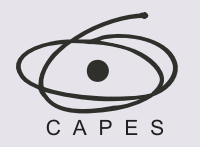

Ministério da Educação

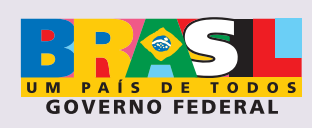

DTFAPESP

Institutional Sponsors

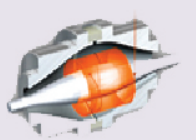

† SHIMADZu UNICAMP

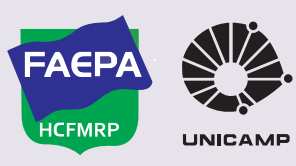

lore High - Performance MS Orbitrap Technology In Proteomics \& Metabolomics analitica Thermo 


\title{
Analysis of acylcarnitine profiles in umbilical cord blood and during the early neonatal period by electrospray ionization tandem mass spectrometry
}

\author{
E. Vieira Neto ${ }^{1,2}$, A.A. Fonseca ${ }^{2}$, R.F. Almeida ${ }^{2}$, M.P. Figueiredo ${ }^{3}$, \\ M.A.S. Porto ${ }^{3}$ and M.G. Ribeiro ${ }^{1}$ \\ ${ }^{1}$ Serviço de Genética Médica, Instituto de Puericultura e Pediatria Martagão Gesteira, \\ Universidade Federal do Rio de Janeiro, Rio de Janeiro, RJ, Brasil \\ 2Laboratório Diagnósticos Laboratoriais Especializados, Rio de Janeiro, RJ, Brasil \\ ${ }^{3}$ Maternidade Escola, Universidade Federal do Rio de Janeiro, Rio de Janeiro, RJ, Brasil
}

\begin{abstract}
Acylcarnitine profiling by electrospray ionization tandem mass spectrometry (ESI-MS/MS) is a potent tool for the diagnosis and screening of fatty acid oxidation and organic acid disorders. Few studies have analyzed free carnitine and acylcarnitines in dried blood spots (DBS) of umbilical cord blood (CB) and the postnatal changes in the concentrations of these analytes. We have investigated these metabolites in healthy exclusively breastfed neonates and examined possible effects of birth weight and gestational age. DBS of CB were collected from 162 adequate for gestational age neonates. Paired DBS of heel-prick blood were collected 4-8 days after birth from 106 of these neonates, the majority exclusively breastfed. Methanol extracts of DBS with deuterium-labeled internal standards were derivatized before analysis by ESI-MS/MS. Most of the analytes were measured using a full-scan method. The levels of the major long-chain acylcarnitines, palmitoylcarnitine, stearoylcarnitine, and oleoylcarnitine, increased by 27, 12, and 109\%, respectively, in the first week of life. Free carnitine and acetylcarnitine had a modest increase: 8 and $11 \%$, respectively. Propionylcarnitine presented a different behavior, decreasing $9 \%$ during the period. The correlations between birth weight or gestational age and the concentrations of the analytes in DBS were weak $(r \leq 0.20)$ or nonsignificant. Adaptation to breast milk as the sole source of nutrients can explain the increase of these metabolites along the early neonatal period. Acylcarnitine profiling in CB should have a role in the early detection of metabolic disorders in high-risk neonates.
\end{abstract}

Key words: Acylcarnitine; Carnitine; Cord blood; Electrospray ionization mass spectrometry; Newborn infants; Reference values

\section{Introduction}

In plasma and in whole blood, total carnitine is present as free and acylcarnitine ester forms. Long-chain acylcarnitine esters are generated intracellularly for the transport of fatty acyl moieties through the mitochondrial inner membrane; in contrast, short- and medium-chain acylcarnitine esters are formed in peroxisomes intended for continuation of oxidation in mitochondria, whereas acylcarnitine esters of various chain lengths are generated in both organelles as a means of modulating the intracellular homeostasis of coenzyme $A(1,2)$.

The determination of free carnitine and acylcarnitine profiles by electrospray ionization tandem mass spectrometry (ESI-MS/MS) in plasma and dried blood spots (DBS) is aided by the fact that both underivatized and butyl esters of acylcarnitines yield a common product ion with a mass of $85 \mathrm{Da}$ (3). Acylcarnitine profiling is a potent tool for the diagnosis and neonatal screening of fatty acid oxidation and organic acid metabolism disorders (4). Cut-off points for free carnitine and important acylcarnitine esters in DBS have been published in conjunction with evaluations of newborn screening programs (5-7). These cut-off points are tradeoffs between a high detection rate of affected neonates

Correspondence: E. Vieira Neto, Serviço de Genética Médica, Instituto de Puericultura e Pediatria Martagão Gesteira, Rua Bruno Lobo, 50, 3ํandar, 21941-912 Rio de Janeiro, RJ, Brasil. Fax: +55-21-2521-9928. E-mail: eduardo.vieira.neto@terra.com.br 
and an acceptable false-positive rate, and represent very high percentiles (e.g., 99.5th or 99.98th), which do not necessarily indicate reference intervals that can be applied for the diagnosis of symptomatic neonates. Few research investigations have reported more typical percentiles as reference limits (e.g., 5.0th-95.0th) for these metabolites in DBS (8).

Although acylcarnitine profiles for umbilical cord blood (CB) collected on filter paper have the potential to identify infants at risk of disorders with a relatively rapid progression after birth, few studies have established reference ranges for this clinical specimen (8-11). Moreover, significant differences are expected to exist between acylcarnitine profiles for $\mathrm{CB}$ and those for blood collected from newborn infants after birth due to the adaptation from a continuous supply of glucose in utero to a neonatal diet based on breast milk, and consequently to fat as a substantial source of energy $(12,13)$. These peculiarities could limit the value of CB acylcarnitine profiles in the detection of disorders of fatty acid oxidation and organic acid metabolism (11).

In the current study, we established reference intervals for free carnitine and acylcarnitine esters for CB and blood collected during the first few days of life (heel-prick blood, HPB) obtained from term neonates that were exclusively breastfed. The results obtained can be used to infer the effects of breastfeeding on the concentrations of these analytes in the first week of life.

\section{Material and Methods}

\section{Study population and blood sample collection}

CB was collected from 176 healthy neonates, 96 females and 80 males. The majority of the newborn infants (91\%$155 / 171$ ) were delivered by spontaneous vaginal delivery, while caesarean births and forceps-assisted vaginal births accounted for $8(13 / 171)$ and $2 \%(3 / 171)$ of the deliveries, respectively. The mean (range) gestational age at birth was 39 weeks and 4 days ( 35 weeks and 5 days -42 weeks and 1 day); three post-term ( $\geq 42$ weeks) and 6 preterm ( $<37$ weeks) infants, at first incorrectly evaluated as term infants, were excluded from the study. The neonates were categorized as small for gestational age (SGA), average for gestational age (AGA) or large for gestational age (LGA) if their birth weight was equal to or below the 2nd percentile, between the 2nd and the 98th percentiles or equal to or above the 98th percentile, respectively, according to the birth weight percentiles provided in the supplemental data of Oken et al. (14). A total of 163 term neonates were classified as AGA, 2 as SGA, and 2 as LGA. As the term SGA and LGA neonates were also excluded, our sample at the beginning of the study (CB collection) actually comprised 163 AGA term newborn infants. None of these had a diagnosis of birth asphyxia, defined as an Apgar score $\leq 6$ at 5 min. CB samples were taken from vessels of the umbilical cord immediately after delivery and spotted on S\&S Grade
903 filter paper (Whatman, UK), allowed to air dry for at least $4 \mathrm{~h}$ and stored in opaque envelopes at room temperature. As one CB sample was refused due to improper collection, it was possible to analyze 162 CB samples.

A second blood sample was prospectively collected by heel prick on day 4-8 from 106 neonates, and processed as CB samples. At this time, mothers were questioned about feeding practices, which were categorized according to the WHO definitions. Mothers reported exclusive breastfeeding for 99 newborn infants, while seven women stated partial breastfeeding, having introduced infant formula to their newborns.

\section{Ethics approval and informed consent}

The study was approved by the Research Ethics Review Committee of "Instituto de Puericultura e Pediatria Martagão Gesteira, Universidade Federal do Rio de Janeiro - UFRJ", and was performed in accordance with Brazilian Guidelines and Regulating Norms on Research Involving Human Subjects and the Helsinki Declaration of 1975 as revised in 1996. Written informed consent was obtained from the mothers of all newborn infants.

\section{Solvents, reagents and internal standards}

The reagents used included HPLC grade methanol and analytical grade acetic acid from Vetec (Brazil). HPLC grade acetonitrile was purchased from Tedia (USA) and $3 \mathrm{~N} \mathrm{HCl}$ in n-butanol was purchased from Regis Technologies (USA). The deuterium-labeled free carnitine and L-acylcarnitines used as internal standards were purchased from Cambridge Isotope Laboratories (USA).

\section{Sample preparation}

Sample preparation was based on previously reported methods $(5-7,15)$. A single $3-\mathrm{mm}$ diameter DBS (1/8 inch) was placed in a well of a 96 -well microtiter polystyrene plate (Sarstedt, USA), followed by the addition of $90 \mu \mathrm{L}$ of a methanol/water $(3: 1 ; \mathrm{v} / \mathrm{v})$ extraction solution containing the deuterium-labeled internal standards. The concentrations of these standards were as follows: $\left[{ }^{2} \mathrm{H}_{9}\right]$-carnitine ( $\left.{ }^{2} \mathrm{H}_{9}-\mathrm{C} 0\right), 0.76 \mu \mathrm{M} ;\left[{ }^{2} \mathrm{H}_{3}\right]$-acetylcarnitine $\left({ }^{2} \mathrm{H}_{3}-\mathrm{C} 2\right), 0.19$ $\mu \mathrm{M}$; $\left[{ }^{2} \mathrm{H}_{3}\right]$-propionylcarnitine, $\left({ }^{2} \mathrm{H}_{3}-\mathrm{C} 3\right), 0.038 \mu \mathrm{M}$; $\left[{ }^{2} \mathrm{H}_{3}\right]-$ butyrylcarnitine $\left({ }^{2} \mathrm{H}_{3}-\mathrm{C} 4\right), 0.038 \mu \mathrm{M}$; $\left[{ }^{2} \mathrm{H}_{9}\right]$-isovalerylcarnitine ( $\left.{ }^{2} \mathrm{H}_{9}-\mathrm{C} 5\right), 0.038 \mu \mathrm{M}$; $\left[{ }^{2} \mathrm{H}_{3}\right]$-octanoylcarnitine $\left({ }^{2} \mathrm{H}_{3}-\mathrm{C} 8\right)$, $0.038 \mu \mathrm{M} ;\left[{ }^{2} \mathrm{H}_{3}\right]$-myristoylcarnitine $\left({ }^{2} \mathrm{H}_{3}-\mathrm{C} 14\right), 0.038 \mu \mathrm{M}$; $\left[{ }^{2} \mathrm{H}_{3}\right]$-palmitoylcarnitine $\left({ }^{2} \mathrm{H}_{3}-\mathrm{C} 16\right), 0.076 \mu \mathrm{M}$. The microtiter plate was covered with sealing film and incubated for 30 min at $27^{\circ} \mathrm{C}$ with shaking at $750 \mathrm{rpm}$ in an NCS incubator (Perkin Elmer, Finland). After seal removal, aliquots of the sample extracts $(60 \mu \mathrm{L})$ were transferred to a polypropylene microtiter plate (USA Scientific, USA) and evaporated to dryness under synthetic air in a Micro-DS 96 sample concentrator (Porvair Sciences, UK). HCl in n-butanol (50 $\mu \mathrm{L}$ of a $3 \mathrm{~N}$ solution) was added to each sample well. Subsequently, the microtiter plate was resealed and incubated 
for $30 \mathrm{~min}$ at $60^{\circ} \mathrm{C}$ to achieve acylcarnitine derivatization. After the n-butanol derivatization, the solution was again evaporated to dryness in the sample concentrator. Finally, the samples were reconstituted with $75 \mu \mathrm{L}$ acetonitrilewater $(80: 20, \mathrm{v} / \mathrm{v})$ with $0.1 \%$ acetic acid, and the plate was covered with aluminum foil. The plate was then ready for ESI-MS/MS analysis after shaking at $750 \mathrm{rpm}$ for $10 \mathrm{~min}$ at $27^{\circ} \mathrm{C}$. Samples were generally prepared and analyzed within 2-3 days from collection.

\section{Mass spectrometry}

An API 2000 triple-quadrupole tandem mass spectrometer (PE Sciex, Canada) operated in positive ion mode was used for acylcarnitine analysis. The system included also a Perkin Elmer series 200 autosampler and a Perkin Elmer series 200 liquid chromatography pump that were employed to transfer $10 \mu \mathrm{L}$ of each sample directly into the ion spray probe at a solvent flow rate of $70 \mu \mathrm{L} / \mathrm{min}$.

Acylcarnitines were analyzed by the precursor-ion scan of m/z 85 mode and scanning from m/z 200 to 550 . Additionally, free carnitine (C0), acetylcarnitine (C2), and propionylcarnitine (C3) and their respective deuteriumlabeled internal standards were analyzed in the multiple reaction monitoring (MRM) mode as recommended by Chace et al. (15) and by the directions for use of PerkinElmer's NeoGram amino acid and acylcarnitine tandem mass spectrometry kit (Perkin Elmer). The MRM transitions used in the analyses were from the n-butanol derivatized molecular ions of $\mathrm{m} / \mathrm{z} 218,227,260,263,274$, and 277 for C0, ${ }^{2} \mathrm{H}_{9}-\mathrm{C} 0, \mathrm{C} 2,{ }^{2} \mathrm{H}_{3}-\mathrm{C} 2, \mathrm{C} 3$, and ${ }^{2} \mathrm{H}_{3}-\mathrm{C} 3$, respectively, to the product ion of $\mathrm{m} / \mathrm{z} 103$ for $\mathrm{CO}$ and ${ }^{2} \mathrm{H}_{9}-\mathrm{CO}$ and $\mathrm{m} / \mathrm{z} 85$ for the other analytes.

The concentrations of free carnitine and acylcarnitines were calculated with the NeoGram software (Perkin Elmer) by comparing the measured analyte intensities to those of the internal standards. Data on linearity, functional sensitivity, reproducibility, recovery, interference, carryover, and drift for the mass spectrometry method used in this study were presented by the manufacturer to the Food and Drug Administration (FDA), and are available at the agency website [http://www.fda.gov/ohrms/dockets/dockets/04n0482/04n0482-bkg00001-vol1.pdf].

\section{Statistical analysis}

The Shapiro-Wilk test was used to assess whether the distributions of free carnitine and the acylcarnitine esters were normal. The Wilcoxon signed rank test was used to investigate significant differences in the distributions of the analytes in CB and HPB. Correlations between birth weight or gestational age and free carnitine or the various acylcarnitine ester concentrations in CB and HPB were tested by the Spearman correlation test. The level of significance was set at 0.05 in all statistical tests. Statistical analysis was performed using the Stata 9.1 software package (StataCorp, USA).

\section{Results}

\section{Distribution of analyte concentrations}

The distributions of the concentrations of free carnitine and the various acylcarnitines in CB and in HPB were not normal, as indicated by the results of the Shapiro-Wilk W test for normality. The quantitatively major acylcarnitines in CB and HPB were the short-chain acylcarnitines $\mathrm{C} 2$ and $\mathrm{C} 3$, and the long-chain acylcarnitines palmitoylcarnitine (C16), stearoylcarnitine (C18), and oleoylcarnitine (C18:1). The acylcarnitine ester profile of $\mathrm{CB}$ differed slightly from that of HPB of 4- to 8-day-old infants in that the peak for C3 was relatively larger and those for the predominant long-chain acylcarnitines less significant. In addition, the order of concentration for the latter in CB, C16 > C18 > C18:1, differed from that found in HPB, C16 > C18:1 > C18 (see Figure 1).

Box-plots for free carnitine and the predominant acylcarnitines are shown in Figure 2.

\section{Descriptive statistics and reference intervals}

Descriptive statistics and reference intervals in $\mathrm{CB}$ and HPB for free carnitine, short-chain, and medium-chain acylcarnitines are summarized in Table 1. Similar parameters are summarized in Table 2 for long-chain acylcarnitines and the acylcarnitine esters derived from dicarboxylic acids, and in Table 3 for those derived from hydroxylated acids. Considering our sample size, we defined the limits of the reference intervals between the 2.5th and 97.5th percentiles, but the lower 2.5th percentile limit is devoid of clinical interest in most instances, with the exception of free carnitine and C2 for the diagnosis of primary systemic carnitine deficiency (16), and C16 and C18 for the diagnosis of carnitine palmitoyltransferase I (CPT I) deficiency (17).

The levels of the quantitatively major long-chain acylcarnitines (C16, C18, C18:1) increased significantly in the first week of life - increase of the median: $27 \%(P<0.0001)$, $12 \%(P=0.0196)$, and $109 \%(P<0.0001)$, respectively (Table 2). Free carnitine and C2 medians had a modest increase, which was nevertheless statistically significant: $8 \%(P=0.0327)$ and $11 \%(P<0.0001)$, respectively (Table 1). $C 3$ presented a different behavior, decreasing $9 \%(P=$ 0.0022 ) in the period (Table 1).

The differences between the medians of the concentrations in CB and HPB of the majority of quantitatively minor short-chain acylcarnitines (Table 1), medium-chain acylcarnitines (Table 1), quantitatively minor long-chain acylcarnitines (Table 2), and of acylcarnitine esters derived from dicarboxylic (Table 2) and hydroxylated acids (Table 3) were significant when evaluated by Wilcoxon's signed rank test. Similar to the quantitatively major acylcarnitine esters $(C 2$, C3, C16, C18, C18:1), they tended to increase during the first week of life. The exceptions were 3-hydroxyisovalerylcarnitine $(\mathrm{C} 5 \mathrm{OH})$, tiglylcarnitine $(\mathrm{C} 5: 1)$, octanoylcarnitine $(\mathrm{C} 8)$, decadienoylcarnitine (C10:2), tetradecadienoylcarnitine 
A

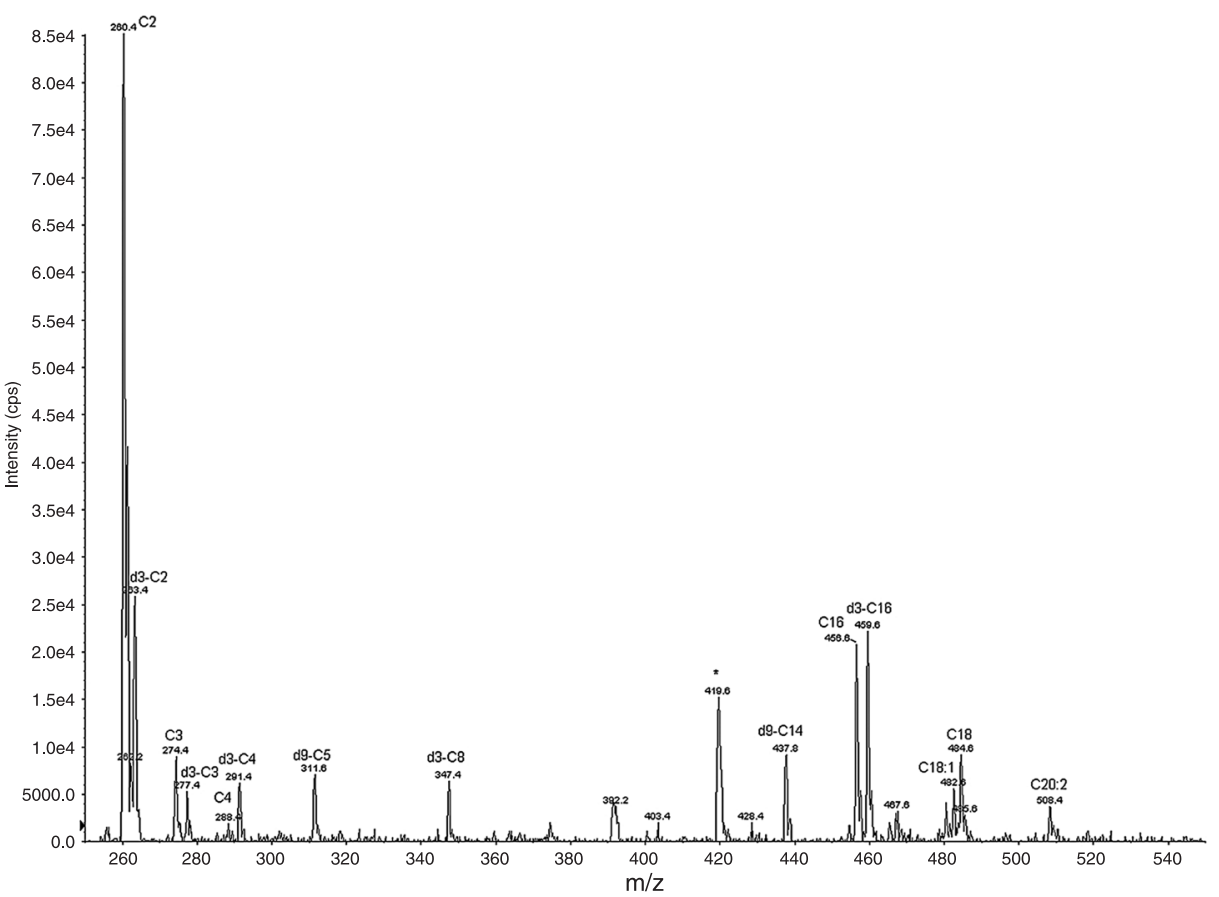

B

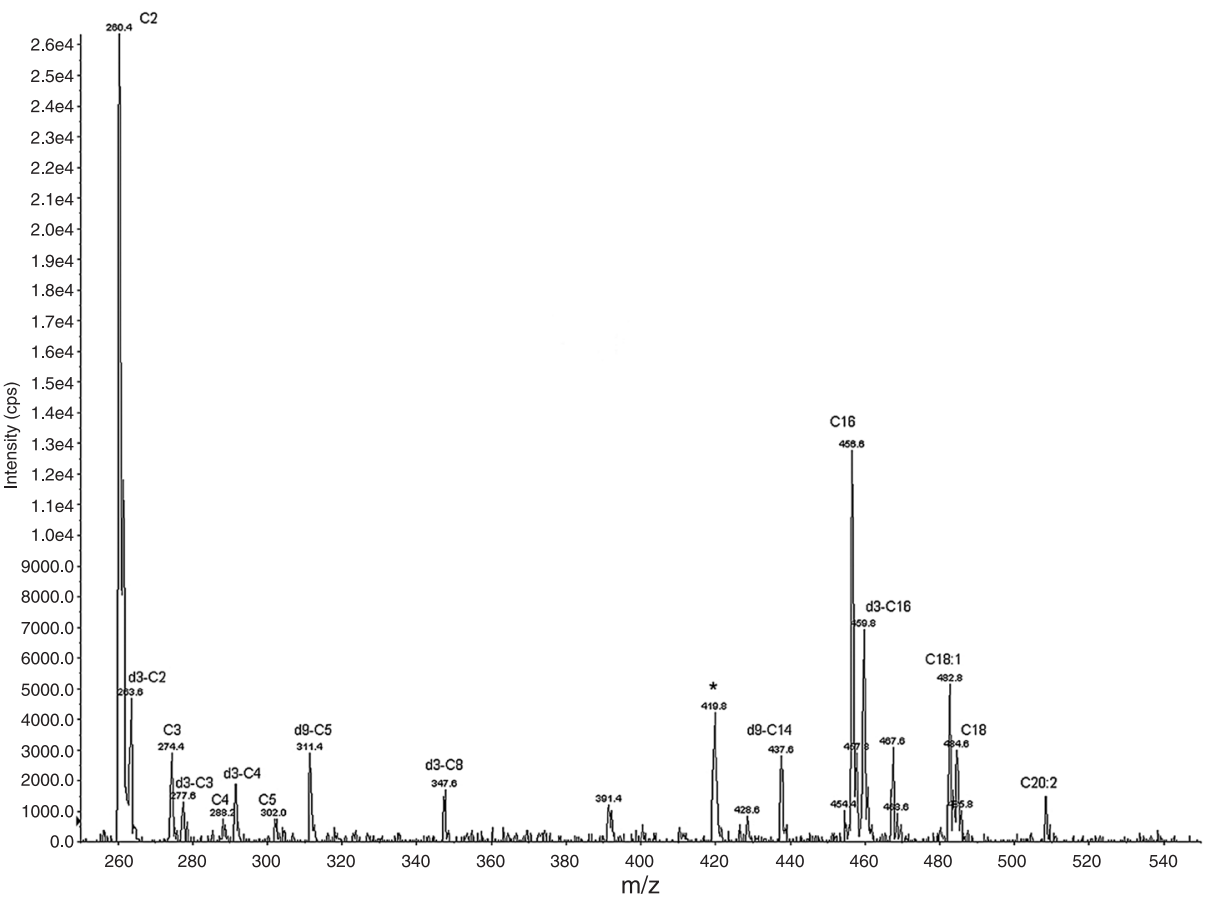

Figure 1. Acylcarnitine ester profiles obtained by a precursor of $\mathrm{m} / \mathrm{z} 85$ scan from dried blood spots (DBS) of a newborn infant. $A$, DBS of a whole cord blood (CB) sample. $B$, DBS of a heel-prick blood (HPB) sample collected on day 5 of life. Note that in HPB the peaks corresponding to long-chain acylcarnitines are more prominent than in CB. Stearoylcarnitine, C18 $(\mathrm{m} / \mathrm{z} 484)$, appears as a larger peak than oleoylcarnitine, C18:1 ( $\mathrm{m} / \mathrm{z} 482)$, in CB. The reverse is true in HPB. The peak for propionylcarnitine, C3 ( $\mathrm{m} / \mathrm{z} 274)$, is usually larger in CB. Stable isotope-labeled acylcarnitine internal standards are identified by the number of deuterium atoms ( $d 3$ or d9). *Artifactual peak. 
(C14:2), 3-hydroxyhexadecenoylcarnitine $(\mathrm{C} 16: 1 \mathrm{OH})$, and linoleylcarnitine (C18:2), whose median concentrations in CB and HPB did not show significant differences.

\section{Correlations between birth weight or gestational age and the analyte concentrations}

For our sample of AGA term newborn infants, the correlations between birth weight or gestational age and the concentrations of free carnitine or the various acylcarnitine esters were very weak $(r \leq 0.20)$ for all analytes in $\mathrm{CB}$, and in most instances the correlations were also nonsignificant, $\mathrm{P}>0.05$ (see supplemental data, Table 1). The exceptions were the significant $(P \leq 0.05)$ albeit very weak correlations found in $\mathrm{CB}$ between birth weight and 3-hydroxybutyrylcarnitine, $\mathrm{C} 4 \mathrm{OH}(r=-0.1689 ; \mathrm{P}=0.0327)$, 3-hydroxytetradecanoylcarnitine, $\mathrm{C} 14 \mathrm{OH}(r=0.1684 ; \mathrm{P}=$ 0.0333 ), and 3-hydroxyoctadecenoylcarnitine, $\mathrm{C} 18: 1 \mathrm{OH}$ $(r=-0.1625 ; \mathrm{P}=0.0333)$, and between gestational age and $\mathrm{C} 4 \mathrm{OH}(r=-0.1602 ; \mathrm{P}=0.0479)$, isovalerylcarnitine, C5 $(r=0.1638 ; \mathrm{P}=0.0430)$, and hexanoylcarnitine, C6 $(r$ $=-0.1912 ; \mathrm{P}=0.0179)$.

In HPB, although the correlations between birth weight or gestational age and the majority of the analytes were also very weak and non-significant (see supplemental data, Table 2), few acylcarnitine ester concentrations in HPB showed weak $(0.20<r \leq 0.40)$ and significant correlations with birth weight as follows: $\mathrm{C} 3(r=0.2099 ; \mathrm{P}=0.0316), \mathrm{C} 4 \mathrm{OH}(r=$ $0.2464 ; \mathrm{P}=0.0113)$, 3-hydroxydodecanoylcarnitine, $\mathrm{C} 12 \mathrm{OH}$ $(r=0.2511 ; \mathrm{P}=0.0098)$, and hexadecenoylcarnitine, $\mathrm{C} 16: 1$ $(r=0.2072 ; \mathrm{P}=0.0340)$. In turn, $\mathrm{C} 14 \mathrm{OH}(r=0.2202 ; \mathrm{P}=$ $0.0277)$ and tetradecadienoylcarnitine, C14:2 ( $r=0.2852$; $P=0.0040$ ), in HPB showed a weak and significant correlation with gestational age.

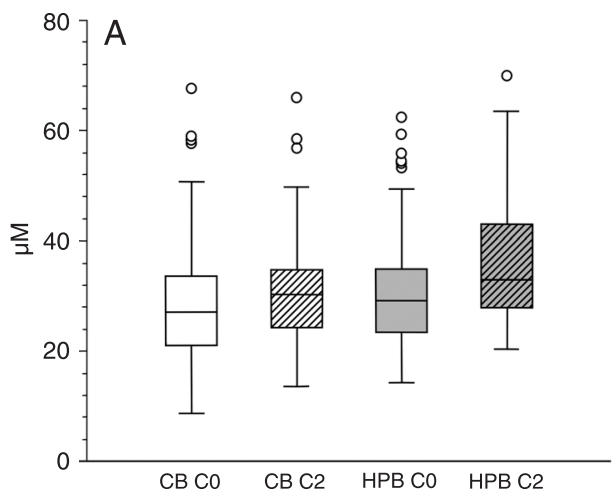

\section{Discussion}

Few studies have described reference limits for free carnitine and acylcarnitine esters in CB by ESI-MS/MS $(8,9,11,18-22)$. In these studies, however, different specimens were used in the analyses. As done in the present study, three of them used DBS of whole CB in order to obtain a full carnitine and acylcarnitine profile $(8,9,11)$. On the other hand, Talián et al. (18) employed dried cord plasma spotted on filter paper cards. Osorio and Pourfarzam (20) favored this specimen for the analysis of total and free carnitine but turned to DBS of whole CB in order to obtain the full acylcarnitine profile (19). Chace et al. (21) compared the results for total and free carnitine in whole cord blood and plasma, both spotted on filter paper. Table 4 compares the concentrations of free carnitine and major acylcarnitines reported by these various investigators to those obtained in the present study.

There is no consensus concerning the clinical specimen (plasma, erythrocytes, or whole blood) that would best reflect the carnitine status of various body compartments of newborn infants, justifying its exclusive recommendation for the detection of eventual carnitine metabolism disorders in this population (23).

Various methods have been reported for the analysis of free carnitine and acylcarnitines in clinical specimens, including fast atom bombardment tandem mass spectrometry (FAB-MS/MS) (24), radioisotopic exchange-highperformance liquid chromatography (RIE-HPLC) (25), and gas chromatography-mass spectrometry (GC-MS) (26). In the case of acylcarnitine profiling by RIE-HPLC, the method exploits the selectivity of carnitine acetyltransferase (CAT) to label acylcarnitines with a radioactive marker. However,

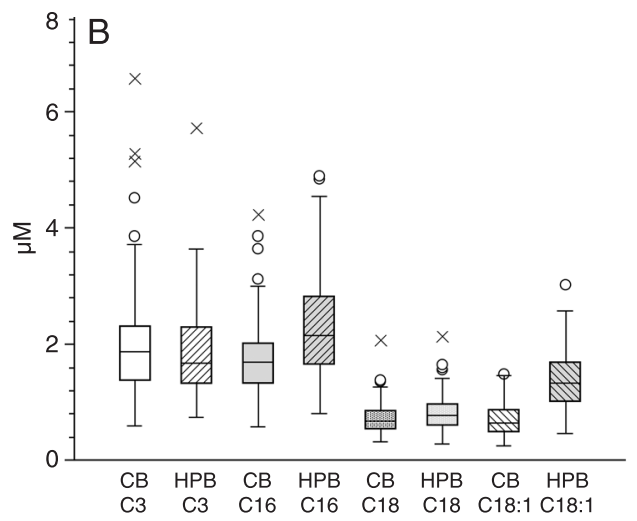

Figure 2. Box-plots of the concentrations of free carnitine and the predominant acylcarnitines in dried blood spots of cord blood and heel-prick blood collected from 4- to 8-day-old newborns. A, Free carnitine (C0) and acetylcarnitine (C2). B, Propionylcarnitine (C3), palmitoylcarnitine (C16), stearoylcarnitine (C18), and oleoylcarnitine (C18:1). The boxes indicate the 1st and 3rd quartiles, and the central line is the median. The ends of the whiskers are 1.5 times the interquartile range below the 1st and above the 3rd quartile. Circles = outliers; $\mathrm{x}=$ extreme outliers $\mathrm{CB}=$ umbilical cord blood; HPB $=$ heel-prick blood sample collected during the first few days of life. 
Table 1. Concentrations of free carnitine and short-chain and medium-chain acylcarnitines in 162 cord blood samples and 106 paired heel-prick blood samples obtained 4-8 days after birth from healthy adequate for gestational age term newborn infants.

\begin{tabular}{|c|c|c|c|}
\hline Analyte & & Cord blood samples & Heel-prick blood samples \\
\hline \multirow[t]{3}{*}{ Free carnitine } & Median & $27.05(25.50-28.70)$ & $29.20(26.41-31.06)^{\star}$ \\
\hline & Range & $8.70-67.50$ & $14.30-62.30$ \\
\hline & 2.5th-97.5th & $14.91-57.08$ & $14.84-56.84$ \\
\hline \multicolumn{4}{|c|}{ Short-chain acylcarnitines } \\
\hline \multirow[t]{3}{*}{$\mathrm{C} 2$} & Median & $30.35(28.10-31.10)$ & $32.95(30.48-35.47)^{\star}$ \\
\hline & Range & $13.60-65.90$ & $20.40-69.80$ \\
\hline & 2.5th-97.5th & $17.51-49.66$ & $21.17-63.20$ \\
\hline \multirow[t]{3}{*}{ C3 } & Median & $1.88(1.69-2.01)$ & $1.68(1.53-1.83)^{*}$ \\
\hline & Range & $0.60-6.57$ & $0.75-5.73$ \\
\hline & 2.5th-97.5th & $0.83-4.46$ & $0.86-3.41$ \\
\hline \multirow[t]{3}{*}{$\mathrm{C} 4$} & Median & $0.25(0.23-0.28)$ & $0.30(0.26-0.34)^{*}$ \\
\hline & Range & $0.05-1.30$ & $0.09-1.20$ \\
\hline & 2.5th-97.5th & $0.09-0.56$ & $0.12-1.03$ \\
\hline \multirow[t]{3}{*}{$\mathrm{C} 5$} & Median & $0.16(0.14-0.18)$ & $0.24(0.21-0.27)^{*}$ \\
\hline & Range & $0.03-0.42$ & $0.07-0.88$ \\
\hline & 2.5th-97.5th & $0.05-0.39$ & $0.07-0.74$ \\
\hline \multirow[t]{3}{*}{ C5:1 } & Median & $0.05(0.05-0.06)$ & $0.06(0.05-0.07)$ \\
\hline & Range & $0.01-0.23$ & $0.02-0.21$ \\
\hline & 2.5th-97.5th & $0.02-0.16$ & $0.02-0.18$ \\
\hline \multicolumn{4}{|c|}{ Medium-chain acylcarnitines } \\
\hline \multirow[t]{3}{*}{ C6 } & Median & $0.08(0.07-0.09)$ & $0.12(0.10-0.13)^{*}$ \\
\hline & Range & $0.02-0.28$ & $0.03-0.34$ \\
\hline & 2.5th-97.5th & $0.02-0.19$ & $0.04-0.32$ \\
\hline \multirow[t]{3}{*}{$\mathrm{C} 8$} & Median & $0.09(0.08-0.11)$ & $0.12(0.10-0.14)$ \\
\hline & Range & $0.02-0.42$ & $0.03-0.40$ \\
\hline & 2.5th-97.5th & $0.03-0.25$ & $0.04-0.36$ \\
\hline \multirow[t]{3}{*}{ C10 } & Median & $0.07(0.06-0.07)$ & $0.10(0.09-0.12)^{*}$ \\
\hline & Range & $0.02-0.20$ & $0.03-0.32$ \\
\hline & 2.5th-97.5th & $0.03-0.18$ & $0.03-0.26$ \\
\hline \multirow[t]{3}{*}{ C10:1 } & Median & $0.08(0.07-0.09)$ & $0.12(0.10-0.13)^{*}$ \\
\hline & Range & $0.02-0.27$ & $0.03-0.36$ \\
\hline & 2.5th-97.5th & $0.03-0.23$ & $0.04-0.30$ \\
\hline \multirow[t]{3}{*}{ C10:2 } & Median & $0.05(0.04-0.05)$ & $0.05(0.05-0.07)$ \\
\hline & Range & $0.02-0.22$ & $0.02-0.16$ \\
\hline & 2.5th-97.5th & $0.03-0.13$ & $0.03-0.16$ \\
\hline \multirow[t]{3}{*}{$\mathrm{C} 12$} & Median & $0.08(0.07-0.08)$ & $0.11(0.09-0.13)^{*}$ \\
\hline & Range & $0.02-0.22$ & $0.03-0.37$ \\
\hline & 2.5th-97.5th & $0.02-0.19$ & $0.04-0.32$ \\
\hline
\end{tabular}

Concentrations are reported in $\mu \mathrm{M}$. Data are reported as median $(95 \% \mathrm{Cl})$, range and 2.5 th and 97.5th percentiles. ${ }^{*} \mathrm{P}<0.05$ compared to cord blood samples (Wilcoxon's signed rank test). 
Table 2. Concentrations of long-chain acylcarnitines and acylcarnitine esters derived from dicarboxylic acids in 162 cord blood samples and 106 paired heel-prick blood samples obtained 4-8 days after birth from healthy adequate for gestational age term newborn infants.

\begin{tabular}{|c|c|c|c|}
\hline Analyte & & Cord blood samples & Heel-prick blood samples \\
\hline \multicolumn{4}{|c|}{ Long-chain acylcarnitines } \\
\hline \multirow[t]{3}{*}{$\mathrm{C} 14$} & Median & $0.14(0.13-0.15)$ & $0.23(0.19-0.25)^{*}$ \\
\hline & Range & $0.02-0.49$ & $0.07-0.50$ \\
\hline & 2.5th-97.5th & $0.05-0.34$ & $0.08-0.43$ \\
\hline \multirow[t]{3}{*}{ C14:1 } & Median & $0.06(0.06-0.07)$ & $0.11(0.09-0.12)^{*}$ \\
\hline & Range & $0.02-0.23$ & $0.02-0.31$ \\
\hline & 2.5th-97.5th & $0.02-0.16$ & $0.02-0.31$ \\
\hline \multirow[t]{3}{*}{ C14:2 } & Median & $0.04(0.03-0.04)$ & $0.05(0.04-0.06)$ \\
\hline & Range & $0.01-0.17$ & $0.02-0.13$ \\
\hline & 2.5th-97.5th & $0.02-0.12$ & $0.02-0.11$ \\
\hline \multirow[t]{3}{*}{ C16 } & Median & $1.70(1.60-1.82)$ & $2.16(2.02-2.43)^{*}$ \\
\hline & Range & $0.58-4.24$ & $0.81-4.89$ \\
\hline & 2.5th-97.5th & $0.63-3.11$ & $0.89-4.64$ \\
\hline \multirow[t]{3}{*}{ C16:1 } & Median & $0.10(0.10-0.12)$ & $0.15(0.13-0.17)^{*}$ \\
\hline & Range & $0.03-0.30$ & $0.02-0.52$ \\
\hline & 2.5th-97.5th & $0.03-0.26$ & $0.04-0.40$ \\
\hline \multirow[t]{3}{*}{ C16:2 } & Median & $0.05(0.05-0.06)$ & $0.06(0.06-0.07)^{*}$ \\
\hline & Range & $0.01-0.19$ & $0.01-0.23$ \\
\hline & 2.5th-97.5th & $0.02-0.15$ & $0.02-0.19$ \\
\hline \multirow[t]{3}{*}{ C18 } & Median & $0.68(0.65-0.72)$ & $0.77(0.69-0.83)^{\star}$ \\
\hline & Range & $0.32-2.07$ & $0.29-2.13$ \\
\hline & 2.5th-97.5th & $0.35-1.34$ & $0.30-1.62$ \\
\hline \multirow[t]{3}{*}{ C18:1 } & Median & $0.65(0.60-0.68)$ & $1.34(1.21-1.41)^{\star}$ \\
\hline & Range & $0.26-1.48$ & $0.47-3.02$ \\
\hline & 2.5th-97.5th & $0.32-1.35$ & $0.57-2.55$ \\
\hline \multirow[t]{3}{*}{ C18:2 } & Median & $0.29(0.26-0.31)$ & $0.31(0.26-0.34)$ \\
\hline & Range & $0.09-0.59$ & $0.07-1.43$ \\
\hline & 2.5th-97.5th & $0.13-0.50$ & $0.11-0.90$ \\
\hline \multicolumn{4}{|c|}{ Acylcarnitine esters derived from dicarboxylic acids } \\
\hline \multirow[t]{3}{*}{ C3DC } & Median & $0.10(0.10-0.12)$ & $0.13(0.10-0.15)^{\star}$ \\
\hline & Range & $0.03-0.48$ & $0.04-0.66$ \\
\hline & 2.5th-97.5th & $0.04-0.35$ & $0.04-0.43$ \\
\hline \multirow[t]{3}{*}{ C4DC } & Median & $0.33(0.30-0.37)$ & $0.39(0.36-0.44)^{*}$ \\
\hline & Range & $0.11-0.99$ & $0.09-1.05$ \\
\hline & 2.5th-97.5th & $0.14-0.76$ & $0.13-0.85$ \\
\hline \multirow[t]{3}{*}{ C5DC } & Median & $0.04(0.04-0.05)$ & $0.06(0.05-0.07)^{*}$ \\
\hline & Range & $0.02-0.15$ & $0.02-0.18$ \\
\hline & 2.5th-97.5th & $0.02-0.12$ & $0.02-0.14$ \\
\hline \multirow[t]{3}{*}{ C6DC } & Median & $0.05(0.04-0.05)$ & $0.06(0.05-0.07)^{*}$ \\
\hline & Range & $0.01-0.23$ & $0.02-0.32$ \\
\hline & 2.5th-97.5th & $0.02-0.14$ & $0.02-0.21$ \\
\hline
\end{tabular}

Concentrations are reported in $\mu \mathrm{M}$. Data are reported as median $(95 \% \mathrm{Cl})$, range and 2.5 th and 97.5th percentiles. ${ }^{*} \mathrm{P}<0.05$ compared to cord blood samples (Wilcoxon's signed rank test). 
long-chain acylcarnitines and dicarboxylic acylcarnitines are not good substrates for CAT, limiting the application of this method (25). Acylcarnitines are charged, polar, and nonvolatile compounds, therefore they are not good candidates for GC-MS techniques, requiring complex derivatization reactions under forced conditions (27).

FAB-MS/MS was introduced by Millington et al. (24) for acylcarnitine detection. This method allowed the metabolic profiling of acylcarnitines from plasma and DBS, as a result of the significant improvement in sensitivity that was achieved (27). Moreover, with the use of continuous flow FAB (CF-FAB), analysis time was significantly reduced. ESI-MS/MS, the method employed in the present study, was initially proposed by Rashed et al. $(27,28)$ as a more robust and sensitive alternative technique to FAB-MS/MS for acylcarnitine profiling in DBS. ESI-MS/MS does not require frequent instrument cleaning and permits higher flow rates than CF-FAB, rendering the method suitable for automation and high sample throughput.

Derivatized ESI-MS/MS assays are employed by the majority of newborn screening laboratories around the world, but it is possible to detect acylcarnitines as their native free acids by underivatized methods. These latter methods have the disadvantage of not differentiating some isobaric acylcarnitines and of eliciting a less intense response from dicarboxylic acid acylcarnitines (29).

It is useful to compare our finding of a significant although modest $8 \%$ increase in free carnitine concentration in the first week of life to the results of other investigators. Meyburg et al. (9) and Cavedon et al. (8) found that free carnitine concentrations remained unchanged in the first days of life by comparing CB samples to paired samples from 5-day-old infants or to unpaired samples from 3- to-6-day-old infants, respectively. In turn, Chace et al. (21) found that free carnitine levels in CB samples from a small cohort of 50 full-term newborns were $48 \%$ lower than the levels in HPB from a large cohort $(N=24,644)$ of $2 \pm 1$-day-old infants. The huge difference in sample size between the two cohorts precludes any further interpretation. Wilcken et al. (16) found a small $(3.4 \%)$ increase in median free carnitine levels in the neonatal population from $26.6 \mu \mathrm{M}$ at day $2(\mathrm{~N}=13,311)$ to $27.5 \mu \mathrm{M}$ at day $5-8(\mathrm{~N}=5.546)$. Therefore, our results agree with the finding from this large-scale study of a small increase in free carnitine levels during the early neonatal period. Table 5 summarizes the findings of the studies that
Table 3. Concentrations of acylcarnitine esters derived from hydroxylated acids in 162 cord blood samples and 106 paired heel-prick blood samples obtained 4-8

\begin{tabular}{|c|c|c|c|}
\hline Analyte & & Cord blood samples & Heel-prick blood samples \\
\hline \multirow[t]{3}{*}{$\mathrm{C} 4 \mathrm{OH}$} & Median & $0.16(0.14-0.18)$ & $0.26(0.21-0.29)^{*}$ \\
\hline & Range & $0.04-0.65$ & $0.04-0.83$ \\
\hline & 2.5th-97.5th & $0.05-0.40$ & $0.08-0.67$ \\
\hline \multirow[t]{3}{*}{$\mathrm{C} 5 \mathrm{OH}$} & Median & $0.18(0.16-0.19)$ & $0.19(0.17-0.20)$ \\
\hline & Range & $0.03-0.70$ & $0.08-0.50$ \\
\hline & 2.5th-97.5th & $0.07-0.39$ & $0.09-0.44$ \\
\hline \multirow[t]{3}{*}{$\mathrm{C} 12 \mathrm{OH}$} & Median & $0.03(0.03-0.04)$ & $0.05(0.04-0.06)^{\star}$ \\
\hline & Range & $0.01-0.11$ & $0.01-0.18$ \\
\hline & 2.5th-97.5th & $0.02-0.09$ & $0.02-0.15$ \\
\hline \multirow[t]{3}{*}{$\mathrm{C} 14 \mathrm{OH}$} & Median & $0.04(0.03-0.04)$ & $0.04(0.04-0.05)^{*}$ \\
\hline & Range & $0.01-0.13$ & $0.02-0.13$ \\
\hline & 2.5th-97.5th & $0.02-0.09$ & $0.02-0.11$ \\
\hline \multirow[t]{3}{*}{$\mathrm{C} 16 \mathrm{OH}$} & Median & $0.04(0.03-0.04)$ & $0.05(0.04-0.06)^{*}$ \\
\hline & Range & $0.01-0.12$ & $0.01-0.15$ \\
\hline & 2.5th-97.5th & $0.02-0.09$ & $0.02-0.13$ \\
\hline \multirow[t]{3}{*}{$\mathrm{C} 16: 10 \mathrm{H}$} & Median & $0.07(0.06-0.07)$ & $0.07(0.06-0.08)$ \\
\hline & Range & $0.02-0.18$ & $0.02-0.20$ \\
\hline & 2.5th-97.5th & $0.02-0.16$ & $0.02-0.19$ \\
\hline \multirow[t]{3}{*}{$\mathrm{C} 18 \mathrm{OH}$} & Median & $0.03(0.02-0.03)$ & $0.04(0.03-0.04)^{*}$ \\
\hline & Range & $0.01-0.10$ & $0.01-0.12$ \\
\hline & 2.5th-97.5th & $0.01-0.08$ & $0.02-0.10$ \\
\hline \multirow[t]{3}{*}{ C18:10H } & Median & $0.03(0.03-0.03)$ & $0.04(0.04-0.05)^{\star}$ \\
\hline & Range & $0.01-0.15$ & $0.01-0.19$ \\
\hline & 2.5th-97.5th & $0.01-0.10$ & $0.02-0.11$ \\
\hline \multirow[t]{3}{*}{$\mathrm{C} 18: 2 \mathrm{OH}$} & Median & $0.10(0.09-0.11)$ & $0.13(0.11-0.14)^{*}$ \\
\hline & Range & $0.03-0.32$ & $0.04-0.36$ \\
\hline & 2.5th-97.5th & $0.04-0.23$ & $0.04-0.26$ \\
\hline
\end{tabular}

Concentrations are reported in $\mu \mathrm{M}$. Data are reported as median $(95 \% \mathrm{Cl})$, range and 2.5th and 97.5th percentiles. ${ }^{*} \mathrm{P}<0.05$ compared to cord blood samples (Wilcoxon's signed rank test).

compared the concentrations of free carnitine for at least two time points during the early neonatal period.

Possibly the fraction of exclusively breastfed newborn infants has a great impact on free carnitine levels during this period, since there is an increased carnitine concentration in human milk during the first two weeks after delivery (30) and the bioavailability of human milk carnitine is superior to that of cow milk or cow milk protein-based infant formula $(12,31)$.

In contrast, similar to the studies of Meyburg et al. (9) and Cavedon et al. (8), we found lower concentrations of almost all acylcarnitines in CB when compared to HPB obtained from the same newborn infants when they were 
Table 4. Concentrations of free carnitine and major acylcarnitines in cord blood samples reported in the literature compared with those obtained in the present study.

\begin{tabular}{|c|c|c|c|c|c|c|c|c|}
\hline \multirow[t]{2}{*}{ Reference } & \multirow[t]{2}{*}{$\mathrm{N}$} & \multirow[t]{2}{*}{ Sample } & \multicolumn{6}{|c|}{ Analyte (mean \pm SD, $1.96 \mathrm{SD}$ or SEM in $\mu \mathrm{M}$, see legend) } \\
\hline & & & $\mathrm{CO}$ & $\mathrm{C} 2$ & C3 & C16 & C18 & C18:1 \\
\hline Present study & 162 & A & $28.47 \pm 9.79$ & $30.75 \pm 8.49$ & $1.98 \pm 0.87$ & $1.73 \pm 0.60$ & $0.73 \pm 0.26$ & $0.69 \pm 0.25$ \\
\hline Meyburg et al. (9) & 70 & A & $31.60 \pm 10.20$ & $10.30 \pm 3.10$ & $2.00 \pm 0.60$ & $2.60 \pm 0.80$ & $1.30 \pm 0.40$ & $1.10 \pm 0.40$ \\
\hline Osorio and Pourfarzam (19) & 130 & A & - & $13.79 \pm 4.61$ & $1.51 \pm 0.64$ & $2.63 \pm 1.30$ & $0.89 \pm 0.41$ & $0.82 \pm 0.38$ \\
\hline Cavedon et al. (8) & 67 & $A$ & $29.10 \pm 2.35$ & $31.80 \pm 1.75$ & $3.50 \pm 0.25$ & $2.90 \pm 0.20$ & - & $1.15 \pm 0.13$ \\
\hline Honzík et al. (22) & 33 & $A$ & $46.87 \pm 13.90$ & - & - & - & - & - \\
\hline \multirow[t]{2}{*}{ Chace et al. (21) } & 50 & A & $21.59 \pm 10.52$ & - & - & - & - & - \\
\hline & & B & $12.95 \pm 4.85$ & - & - & - & - & - \\
\hline Osorio and Pourfarzam (20) & 130 & $\mathrm{~B}$ & $18.50 \pm 3.40$ & - & - & - & - & - \\
\hline Talián et al. (18) & 20 & B & $20.00 \pm 1.30$ & $11.24 \pm 1.56$ & $0.22 \pm 0.02$ & $0.15 \pm 0.01$ & $0.07 \pm 0.01$ & $0.12 \pm 0.01$ \\
\hline \multirow[t]{2}{*}{ Reference } & $\mathrm{N}$ & Sample & \multicolumn{6}{|c|}{ Analyte (95\% reference limits in $\mu \mathrm{M})$} \\
\hline & & & $\mathrm{CO}$ & $\mathrm{C} 2$ & C3 & C16 & C18 & C18.1 \\
\hline Present study & 162 & $A$ & 14.91-57.08 & $17.51-49.66$ & $0.83-4.46$ & $0.63-3.11$ & $0.35-1.34$ & $0.32-1.35$ \\
\hline Walter et al. (11) & 12,952 & $A$ & $14.41-57.91$ & - & $0.79-3.44$ & $0.87-4.81$ & $0.23-1.80$ & $0.29-1.84$ \\
\hline
\end{tabular}

The data for most studies, including the present one, are reported as means $\pm S D$, while for Cavedon et al. (8) they are means \pm 1.96 SD $(95 \%$ confidence interval), and for Talián et al. (18), means \pm SEM. $N=$ total number of samples. $A=$ whole cord blood spotted on filter paper; $B=$ cord blood plasma spotted on filter paper.

Table 5. Variations in the concentrations of free carnitine detected in studies that compared at least two time points during the early neonatal period.

\begin{tabular}{lccccc}
\hline Reference & $\mathrm{N}$ & Cord blood & 2nd sample (age in days) & $\mathrm{N}$ & Heel-prick blood \\
\hline Present study & 162 & $27.05(15.73-47.27)^{\ddagger}$ & $4-8$ & 106 & $29.20(15.91-53.72)^{\ddagger \dagger}$ \\
Meyburg et al. (9) & 70 & $31.60 \pm 10.20 \S$ & 5 & 70 & $30.60 \pm 10.60 \S$ \\
Wilcken et al. (16) & 13,311 & $26.60(14.00-52.00)^{\text {ał }}$ & $5-8$ & 5,546 & $27.50(14.30-49.60)^{\ddagger \dagger \dagger}$ \\
Chace et al. (21) & 50 & $21.59 \pm 10.52 \S$ & $2 \pm 1 \S$ & 24,644 & $46.85 \pm 21.19 \S+\dagger$ \\
Cavedon et al. (8) & 67 & $29.10(26.80-31.50)^{\Uparrow}$ & $3-6$ & 117 & $29.40(27.60-31.20)^{\Uparrow}$ \\
\hline
\end{tabular}

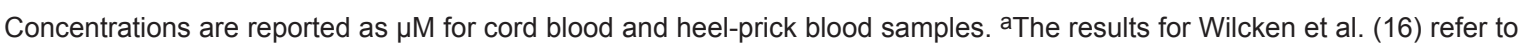
heel-prick blood samples collected on the second day of life. Age = age of the neonates in days at the time of the second matched sample collection for the present study and that of Meyburg et al. (9). For the other studies, the second samples were unmatched and obtained from another group of neonates at the age shown in the table. $¥$ Median (2.5th and 97.5th percentiles). §Means \pm SD. TMeans $\pm 1.96 \mathrm{SD}\left(95 \%\right.$ confidence interval). ${ }^{\mathrm{T}} \mathrm{P}<0.05$ for differences between the medians (present study). The differences between the means of the concentrations of the two samples were not significant in Cavedon et al. (8) and Meyburg et al. (9). ${ }^{\dagger}+$ Significance test for the differences observed was not performed.

4 to 8 days old (paired samples, see Figures 1 and 2). This finding could be attributed to the low plasma lipid levels at birth due to a limited fatty acid transfer across the human placenta (32). Moreover, the increase in the levels of several acylcarnitines in the early neonatal period is a consequence of the process of adaptation to breast milk as the sole source of nutrients and energy, with its large concentration of fatty acids, primarily derived from triglycerides, which account for $35-50 \%$ of the total daily energy supply of the newborn infant (33). Further support of this assumption is the fact that the predominant long-chain acylcarnitines in the early neonatal period, C16 and especially C18:1 (see Figures 1 and 2), are the acylcarnitine esters of the quantitatively major fatty acids in breast milk, palmitic (16:0) and oleic 


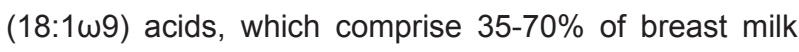
total fatty acids (34). Conversely, the quantitatively major acylcarnitines in CB, C16 and C18 (see Figures 1 and 2), correspond to the predominance of palmitic (16:0) and stearic (18:0) acids in the fraction of nonesterified fatty acids in maternal blood and CB (32).

Propionylcarnitine (C3) behaved differently as its median concentration in $\mathrm{CB}$ was significantly higher than that in HPB (see Figure 2). Cavedon et al. (8) obtained similar results, although comparing unpaired samples. Meyburg et al. (9), in contrast, showed an increase in mean C3 concentration in paired HPB samples from 5-day-old newborn infants when compared to CB samples. Walter et al. (11), in a large-scale cohort study, found many newborn infants with very high C3 levels in CB that decreased to normal levels when a second newborn screening sample was collected. We also observed the presence of extreme outliers of C3 concentration in CB samples (see Figure 2).

There are some case series reports indicating the clinical utility of free carnitine and acylcarnitine ester profile in $\mathrm{CB}$ for the selective screening of high-risk newborn infants immediately after birth. Patterson et al. (35) reported a case of isovaleric acidemia and two cases of medium-chain acyl-coenzyme A dehydrogenase deficiency (MCADD) in newborn infants with a positive family history that were diagnosed by abnormalities in relevant acylcarnitine species in CB. They also ruled out the diagnosis of MCADD in four other high-risk newborn infants whose C8 levels in CB were found to be normal. Nassogne et al. (36) reported the diagnosis of CPT II deficiency in a newborn sibling of an affected proband by an acylcarnitine ester profile of $C B$. An increase of long-chain acylcarnitines from C12 to C18 was demonstrated in CB while the organic acid profile of the first urine samples did not show any alteration. Isackson et al. (37) described a newborn infant along with other four patients, whose prior sibling had died at birth, who presented significant elevations in long-chain CB acylcarnitines and a clinical course consistent with the severe, lethal neonatal form of CPT II deficiency, confirmed by CPT II assay in cultured skin fibroblasts and genomic DNA analysis. Yokoi et al. (38) reported a Japanese neonate with holocarboxylase

\section{References}

1. Ramsay RR, Gandour RD, van der Leij FR. Molecular enzymology of carnitine transfer and transport. Biochim Biophys Acta 2001; 1546: 21-43.

2. Steiber A, Kerner J, Hoppel CL. Carnitine: a nutritional, biosynthetic, and functional perspective. Mol Aspects Med 2004; 25: 455-473.

3. Chace DH, Kalas TA, Naylor EW. The application of tandem mass spectrometry to neonatal screening for inherited disorders of intermediary metabolism. Annu Rev Genomics Hum Genet 2002; 3: 17-45.

4. Carpenter KH, Wiley V. Application of tandem mass spectrom- synthetase deficiency and a positive family history who had received prenatal administration of biotin from 33 weeks' gestation and whose serum cord blood sample showed elevated $\mathrm{C} 3$ and $\mathrm{C} 5 \mathrm{OH}$ levels.

Since CB can reflect changes in maternal blood rather than the baby's situation, acylcarnitine analysis in CB has revealed maternal metabolic disease in completely asymptomatic or vaguely symptomatic mothers $(10,11)$. Vijay et al. (10) reported the identification of four unrelated women from consanguineous families of Asian origin discovered to have primary systemic carnitine deficiency as a consequence of finding low free carnitine concentrations in CB or HPB samples from their infants. Santos et al. (39) diagnosed MCADD in a woman who presented acute fatty liver at the 39th week of pregnancy and whose unaffected infant was identified with high C8 levels in CB. Interestingly, in both reports the abnormal results were initially attributed to metabolic disorders affecting the newborn infants, but the detection of normal profiles in follow-up samples made the authors turn their attention to the babies' mothers.

These reports demonstrate that the collection of CB at the time of delivery for the analysis of free carnitine and acylcarnitines can be appropriate for the selective screening of high-risk newborn infants, including those with a family history of affected siblings, perinatal or neonatal death, or who are offspring of consanguineous marriages or of mothers affected by acute fatty liver. This is especially true in the Brazilian scenario where newborn screening, although currently established to be undertaken at 3-7 days, shows a poor timeliness in many Brazilian states (40), which is unacceptable for the purpose of selective screening of high-risk newborn infants.

\section{Acknowledgments}

The authors thank Mr. Victor R. De Jesus from the Centers for Disease Control and Prevention, Atlanta, for revising the English language of the manuscript, Ms. Cecilia Pessanha Lima for valuable guidance with the statistical analyses and Mr. Osman L. Perdigão for technical support in sample preparation and mass spectrometry analysis. etry to biochemical genetics and newborn screening. Clin Chim Acta 2002; 322: 1-10.

5. Zytkovicz TH, Fitzgerald EF, Marsden D, Larson CA, Shih VE, Johnson DM, et al. Tandem mass spectrometric analysis for amino, organic, and fatty acid disorders in newborn dried blood spots: a two-year summary from the New England Newborn Screening Program. Clin Chem 2001; 47: 1945-1955.

6. Schulze A, Lindner M, Kohlmuller D, Olgemoller K, Mayatepek E, Hoffmann GF. Expanded newborn screening for inborn errors of metabolism by electrospray ionization-tandem mass spectrometry: results, outcome, and implications. Pediatrics 
2003; 111: 1399-1406

7. Rashed MS, Bucknall MP, Little D, Awad A, Jacob M, Alamoudi $\mathrm{M}$, et al. Screening blood spots for inborn errors of metabolism by electrospray tandem mass spectrometry with a microplate batch process and a computer algorithm for automated flagging of abnormal profiles. Clin Chem 1997; 43: 1129-1141.

8. Cavedon CT, Bourdoux P, Mertens K, Van Thi HV, Herremans $\mathrm{N}$, de Laet $\mathrm{C}$, et al. Age-related variations in acylcarnitine and free carnitine concentrations measured by tandem mass spectrometry. Clin Chem 2005; 51: 745-752.

9. Meyburg J, Schulze A, Kohlmueller D, Linderkamp O, Mayatepek E. Postnatal changes in neonatal acylcarnitine profile. Pediatr Res 2001; 49: 125-129.

10. Vijay S, Patterson A, Olpin S, Henderson MJ, Clark S, Day C, et al. Carnitine transporter defect: diagnosis in asymptomatic adult women following analysis of acylcarnitines in their newborn infants. J Inherit Metab Dis 2006; 29: 627-630.

11. Walter JH, Patterson A, Till J, Besley GT, Fleming G, Henderson MJ. Bloodspot acylcarnitine and amino acid analysis in cord blood samples: efficacy and reference data from a large cohort study. J Inherit Metab Dis 2009; 32: 95-101.

12. de Rooy L, Hawdon J. Nutritional factors that affect the postnatal metabolic adaptation of full-term small- and large-for-gestational-age infants. Pediatrics 2002; 109: E42.

13. Girard J. Metabolic adaptations to change of nutrition at birth. Biol Neonate 1990; 58 (Suppl 1): 3-15.

14. Oken E, Kleinman KP, Rich-Edwards J, Gillman MW. A nearly continuous measure of birth weight for gestational age using a United States national reference. BMC Pediatr 2003; 3: 6.

15. Chace DH, DiPerna JC, Mitchell BL, Sgroi B, Hofman LF, Naylor EW. Electrospray tandem mass spectrometry for analysis of acylcarnitines in dried postmortem blood specimens collected at autopsy from infants with unexplained cause of death. Clin Chem 2001; 47: 1166-1182.

16. Wilcken B, Wiley V, Sim KG, Carpenter K. Carnitine transporter defect diagnosed by newborn screening with electrospray tandem mass spectrometry. J Pediatr 2001; 138: 581-584.

17. Fingerhut R, Roschinger W, Muntau AC, Dame T, Kreischer J, Arnecke R, et al. Hepatic carnitine palmitoyltransferase I deficiency: acylcarnitine profiles in blood spots are highly specific. Clin Chem 2001; 47: 1763-1768.

18. Talián GC, Komlósi K, Decsi T, Koletzko B, Melegh B. Determination of carnitine ester patterns during the second half of pregnancy, at delivery, and in neonatal cord blood by tandem mass spectrometry: complex and dynamic involvement of carnitine in the intermediary metabolism. Pediatr Res 2007; 62: 88-92.

19. Osorio $\mathrm{JH}$, Pourfarzam M. [Early diagnosis of neurometabolic diseases by tandem mass spectrometry. Acylcarnitine profile from cord blood]. Rev Neurol 2004; 38: 11-16.

20. Osorio JH, Pourfarzam M. Carnitina libre y total en sangre de cordón umbilical. Rev Colomb Obstet Ginecol 2002; 53: 335340 .

21. Chace DH, Pons R, Chiriboga CA, McMahon DJ, Tein I, Naylor EW, et al. Neonatal blood carnitine concentrations: normative data by electrospray tandem mass spectometry. Pediatr Res 2003; 53: 823-829.

22. Honzík T, Chrastina R, Hansíková H, Böhm M, Martincová O, Plavka R, et al. Carnitine concentrations in term and preterm newborns at birth and during the first days of life. Prague Med Rep 2005; 106: 297-306.

23. Crill CM, Helms RA. The use of carnitine in pediatric nutrition.
Nutr Clin Pract 2007: 22: 204-213.

24. Millington DS, Norwood DL, Kodo N, Roe CR, Inoue F. Application of fast atom bombardment with tandem mass spectrometry and liquid chromatography/mass spectrometry to the analysis of acylcarnitines in human urine, blood, and tissue. Anal Biochem 1989; 180: 331-339.

25. Schmidt-Sommerfeld E, Zhang L, Bobrowski PJ, Penn D. Quantitation of short- and medium-chain acylcarnitines in plasma by radioisotopic exchange/high-performance liquid chromatography. Anal Biochem 1995; 231: 27-33.

26. Lowes S, Rose ME, Mills GA, Pollitt RJ. Identification of urinary acylcarnitines using gas chromatography-mass spectrometry: preliminary clinical applications. J Chromatogr 1992; 577: 205214.

27. Rashed MS, Ozand PT, Harrison ME, Evans S. Electrospray tandem mass spectrometry in the diagnosis of organic acidemias. Rapid Commun Mass Spectrom 1994; 8: 129-133.

28. Rashed MS, Ozand PT, Bucknall MP, Little D. Diagnosis of inborn errors of metabolism from blood spots by acylcarnitines and amino acids profiling using automated electrospray tandem mass spectrometry. Pediatr Res 1995; 38: 324-331.

29. De Jesus V, Chace DH, Lim TH, Mei JV, Hannon WH. Comparison of amino acids and acylcarnitines assay methods used in newborn screening assays by tandem mass spectrometry. Clin Chim Acta 2010; 411: 684-689.

30. Giovannini M, Agostoni C, Salari PC. Is carnitine essential in children? J Int Med Res 1991; 19: 88-102.

31. Warshaw JB, Curry E. Comparison of serum carnitine and ketone body concentrations in breast- and in formula-fed newborn infants. J Pediatr 1980; 97: 122-125.

32. Berghaus TM, Demmelmair H, Koletzko B. Fatty acid composition of lipid classes in maternal and cord plasma at birth. Eur $J$ Pediatr 1998; 157: 763-768.

33. Akre J. Infant feeding. The physiological basis. Bull World Health Organ 1989; 67 (Suppl): 1-108.

34. Smit EN. Essential fatty acid deficiency in malnourished children: erythrocyte and breastmilk fatty acid compositions in different populations. [Doctoral thesis]. Groningen: University of Groningen, The Netherlands; 2002.

35. Patterson AL, Pourfarzam M, Henderson MJ. The utility of cord blood analysis in the diagnosis of organic acidaemias. $J$ Inherit Metab Dis 2000; 23 (Suppl 1): 84 (Abstract).

36. Nassogne MC, Jakobs C, Brivet M, Sharrard M, Garcia A, Rabier D, et al. Evolution of plasma acylcarnitines pattern in a newborn with prenatal diagnosis of CPT II deficiency: evidence of improvement upon carnitine supplementation. $J$ Inherit Metab Dis 2000; 23 (Suppl 1): 116 (Abstract).

37. Isackson $\mathrm{PJ}$, Bennett MJ, Lichter-Konecki $\mathrm{U}$, Willis $\mathrm{M}$, Nyhan WL, Sutton VR, et al. CPT2 gene mutations resulting in lethal neonatal or severe infantile carnitine palmitoyltransferase II deficiency. Mol Genet Metab 2008; 94: 422-427.

38. Yokoi K, Ito T, Maeda Y, Nakajima Y, Kurono Y, Sugiyama N, et al. A case of holocarboxylase synthetase deficiency with insufficient response to prenatal biotin therapy. Brain Dev 2009; 31: 775-778.

39. Santos L, Patterson A, Moreea SM, Lippiatt CM, Walter J, Henderson M. Acute liver failure in pregnancy associated with maternal MCAD deficiency. J Inherit Metab Dis 2007; 30: 103.

40. Botler J, Camacho LA, da Cruz MM, George P. [Neonatal screening - the challenge of an universal and effective coverage]. Cien Saude Colet 2010; 15: 493-508. 